(C) 2007 IEEE. Personal use of this material is permitted. Permission from IEEE must be obtained for all other uses, in any current or future media, including reprinting/republishing this material for advertising or promotional purposes, creating new collective works, for resale or redistribution to servers or lists, or reuse of any copyrighted component of this work in other works. 


\title{
An improved Phase Variable Model Based on Electro-magnetic Field Coupled with its External Circuits for Performance Evaluation of Permanent Magnet Brushless DC Motors
}

\author{
Jiaxin Chen ${ }^{1}$, Youguang $\mathrm{Guo}^{2}$, Jianguo Zhu ${ }^{2}$ \\ ${ }^{1}$ College of Electromechanical Engineering, Donghua University, Shanghai, China. chjiaxin@dhu.edu.cn \\ ${ }^{2}$ Faculty of Engineering, University of Technology, Sydney, NSW, Australia. youguang@eng.uts.edu.au, joe@eng.uts.edu.au
}

\begin{abstract}
This paper presents the comprehensive performance evaluation of a brushless permanent magnet (PM) DC (BLDC) motor in dynamic conditions by an improved phase variable model based on electro-magnetic field coupled with its external circuits. In the proposed model, the inductances, back electromotive force (emf) and cogging torque are obtained by nonlinear finite element analysis (FEA). The phase variable model is built and implemented in the MATLAB/Simulink through a method of look-up table to decide the emf which depends on rotor position. Further more, based on a mathematical function for the decision to the voltage of the three phase winding central point, the model could not only solve the problem to decide the input voltage of the phase which is in a non-energized condition, and also make the transient current process during the commutation clear. The theoretical verification is also given in detail. By using the developed model, the comprehensive performance of BLDC motors could be investigated.
\end{abstract}

\section{INTRODUCTION}

Thanks to their advantages such as high efficiency, high power density and high drive performance, permanent magnet (PM) brushless DC (BLDC) motors were used in wide applications in industrial and domestic appliance in the past few decades [1]. As a very important part in electrical driving system, a fast and accurate model for predicting, assessing and optimizing the performance of BLDC motors would be always useful.

For dynamic performance evaluation, compared with an equivalent electric circuit model, the time-stepping nonlinear finite element analysis (FEA) procedure can give accurate results but is more time consuming [2]. A phase variable model of BLDC motor based on FEA and coupled with external circuits, which behaves much faster with the same level of accuracy, has been introduced and verified in [3]. In this model, the inductances, back electromotive force (emf) and cogging torque were obtained by nonlinear FEA, and the problem that the equation-based model cannot be applied to BLDC directly was solved by a method of using a model composed of several circuit components indirectly. Here, a method of pure mathematic approach is proposed. By using this method, the voltage of the $\mathrm{Y}$-type central point of three phase windings can be achieved, the equation-based model of BLDC motor can be applied to BLDC simulation system directly. The theoretical verification is also given in detail.

As a model for dynamic performance evaluation of PM BLDC motor specially, the functions of the model must be considered along with the special requirements.

\section{EQUATION-BASED PHASE VARIABLE MODEL}

The equation-based phase variable model of BLDC motor is given as:

$$
\begin{aligned}
& V_{a b c}=r_{a b c} i_{a b c}+\frac{d \psi_{a b c}}{d t}+e_{a b c} \\
& \psi_{a b c}=L_{a b c} i_{a b c} \\
& T_{m}=\frac{e_{a} i_{a}+e_{b} i_{b}+e_{c} i_{c}}{\omega_{r}}+T_{c o g} \\
& J \frac{d \omega_{r}}{d t}=T_{m}-B \omega_{r}-T_{L}
\end{aligned}
$$

$\left[\begin{array}{c}\frac{d \Psi_{s a}}{d t} \\ \frac{d \Psi_{s b}}{d t} \\ \frac{d \Psi_{s c}}{d t}\end{array}\right]=\left[\begin{array}{ccc}\frac{\partial \Psi_{s a}}{\partial i_{a}} & \frac{\partial \Psi_{s a}}{\partial i_{b}} & \frac{\partial \Psi_{s a}}{\partial i_{c}} \\ \frac{\partial \Psi_{s b}}{\partial i_{b}} & \frac{\partial \Psi_{s b}}{\partial i_{b}} & \frac{\partial \Psi_{s b}}{\partial i_{a}} \\ \frac{\partial \Psi_{s c}}{\partial i_{a}} & \frac{\partial \Psi_{s c}}{\partial i_{b}} & \frac{\partial \Psi_{s c}}{\partial i_{c}}\end{array}\right]\left[\begin{array}{c}\frac{d i_{a}}{d t} \\ \frac{d i_{b}}{d t} \\ \frac{d i_{c}}{d t}\end{array}\right]+\left[\begin{array}{c}\frac{\partial \Psi_{s a}}{\partial \theta} \\ \frac{\partial \Psi_{s b}}{\partial \theta} \\ \frac{\partial \Psi_{s c}}{\partial \theta}\end{array}\right] \frac{d \theta}{d t}$ $\left[\begin{array}{l}\frac{d \Psi_{s a}}{d t} \\ \frac{d \Psi_{s b}}{d t} \\ \frac{d \Psi_{s c}}{d t}\end{array}\right]=\left[\begin{array}{lll}L_{a a} & L_{a b} & L_{a c} \\ L_{b a} & L_{b b} & L_{b c} \\ L_{c a} & L_{c b} & L_{c c}\end{array}\right]\left[\begin{array}{c}\frac{d i_{a}}{d t} \\ \frac{d i_{b}}{d t} \\ \frac{d i_{c}}{d t}\end{array}\right]$ 


$$
\begin{aligned}
& +\left[\begin{array}{ccc}
\frac{d L_{a a}}{d \theta} & \frac{d L_{a b}}{d \theta} & \frac{d L_{a c}}{d \theta} \\
\frac{d L_{b a}}{d \theta} & \frac{d L_{b b}}{d \theta} & \frac{d L_{b c}}{d \theta} \\
\frac{d L_{c a}}{d \theta} & \frac{d L_{c b}}{d \theta} & \frac{d L_{c c}}{d \theta}
\end{array}\right]\left[\begin{array}{l}
i_{a} \\
i_{b} \\
i_{c}
\end{array}\right] \omega p \\
& {\left[\begin{array}{l}
v_{a} \\
v_{b} \\
v_{c}
\end{array}\right]=\left[\begin{array}{ccc}
r_{a} & 0 & 0 \\
0 & r_{b} & 0 \\
0 & 0 & r_{c}
\end{array}\right]\left[\begin{array}{l}
i_{a} \\
i_{b} \\
i_{c}
\end{array}\right]+\left[\begin{array}{lll}
L_{a a} & L_{a b} & L_{a c} \\
L_{b a} & L_{b b} & L_{b c} \\
L_{c a} & L_{c b} & L_{c c}
\end{array}\right]\left[\begin{array}{c}
\frac{d i_{a}}{d t} \\
\frac{d i_{b}}{d t} \\
\frac{d i_{c}}{d t}
\end{array}\right]} \\
& +\left[\begin{array}{ccc}
\frac{d L_{a a}}{d \theta} & \frac{d L_{a b}}{d \theta} & \frac{d L_{a c}}{d \theta} \\
\frac{d L_{b a}}{d \theta} & \frac{d L_{b b}}{d \theta} & \frac{d L_{b c}}{d \theta} \\
\frac{d L_{c a}}{d \theta} & \frac{d L_{c b}}{d \theta} & \frac{d L_{c c}}{d \theta}
\end{array}\right]\left[\begin{array}{c}
i_{a} \\
i_{b} \\
i_{c}
\end{array}\right] \omega p+\left[\begin{array}{c}
e_{a}(\theta) \\
e_{b}(\theta) \\
e_{c}(\theta)
\end{array}\right] \\
& L_{a b}=L_{b a}, L_{b c}=L_{c b}, L_{c a}=L_{a c}, r_{a}=r_{b}=r_{c} \\
& i_{a}+i_{b}+i_{c}=0
\end{aligned}
$$

$$
\begin{array}{r}
+\frac{\left[U_{b}-\left(L_{a b}+L_{b b}+L_{c b}\right) \frac{d i_{b}}{d t}-\frac{d\left(L_{a b}+L_{b b}+L_{c b}\right) i_{b} \omega}{d \theta}\right]}{3} \\
+\frac{\left[U_{c}-\left(L_{a c}+L_{b c}+L_{c c}\right) \frac{d i_{c}}{d t}-\frac{d\left(L_{a c}+L_{b c}+L_{c c}\right) i_{c} \omega}{d \theta}\right]}{3} \\
+\frac{\left[e_{a}(\theta)+e_{b}(\theta)+e_{c}(\theta)\right]}{3}
\end{array}
$$

The values of $U_{a}, U_{b}$ and $U_{c}$ are decided by the switching state of inverter with three phases, the state of PWM and the phase current of windings. When one phase current, for example, the $i_{a}$ of phase $a$ is zero, and the associated circuit is open-circuited, in other words, the winding of phase $a$ is in a non-energized condition, under the consideration of (8) and (9), the corresponding voltage of input port, $U_{N}$ and $U_{a}$ can be decided by (13) and (14).

All the above variables are used as their conventional meanings. The $L_{a b c}, e_{a b c}$, and $T_{c o g}$ profiles are obtained from the nonlinear transient FE solutions, in which the rotor position dependence and the saturation effect are considered [4-6].

\section{Calculation of Central Point Voltage}

Supposing that the voltage of central point at any time is $U_{N}$, and the voltages of input ports of three phases are $U_{a}, U_{b}$ and $U_{c}$, then one can obtain

$$
U_{a b c}=\left[\begin{array}{c}
U_{a} \\
U_{b} \\
U_{c}
\end{array}\right]=\left[\begin{array}{c}
v_{a} \\
v_{b} \\
v_{c}
\end{array}\right]+\left[\begin{array}{c}
U_{N} \\
U_{N} \\
U_{N}
\end{array}\right]
$$

Then

$$
U_{N}=\frac{\left[\begin{array}{lll}
1 & 1 & 1
\end{array}\right] *\left(U_{a b c}-v_{a b c}\right)}{3}
$$

Substituting (7) into (11) and considering (9), the voltage (11) becomes

$U_{N}=\frac{\left[U_{a}-\left(L_{a a}+L_{b a}+L_{c a}\right) \frac{d i_{a}}{d t}-\frac{d\left(L_{a a}+L_{b a}+L_{c a}\right) i_{a} \omega}{d \theta}\right]}{3}$

$$
\begin{aligned}
U_{N}= & \frac{\left[U_{b}-L_{b b} \frac{d i_{b}}{d t}-\frac{d L_{b b}}{d \theta} i_{b} \omega\right]}{2} \\
& +\frac{\left[U_{c}-L_{c c} \frac{d i_{c}}{d t}-\frac{d L_{c c}}{d \theta} i_{c} \omega\right]}{2}-\frac{\left[e_{b}(\theta)+e_{c}(\theta)\right]}{2}
\end{aligned}
$$

$$
\begin{aligned}
U_{a}= & U_{N}+\left(L_{a a}+L_{b a}+L_{c a}\right) \frac{d i_{a}}{d t}+e_{a}(\theta) \\
& +\left(\frac{d L_{a b}}{d \theta} i_{b}+\frac{d L_{a c}}{d \theta} i_{c}\right) \omega+\left(L_{a b} \frac{d i_{b}}{d t}+L_{a c} \frac{d i_{c}}{d t}\right)
\end{aligned}
$$
under the state of duty-off, the voltage of input port of phase $a$ can be decided by

$$
\begin{aligned}
& \text { if } i_{a}>0 \text {, then } U_{a}=U_{\text {bus }} \\
& \text { if } i_{a}<0 \text {, then } U_{a}=0
\end{aligned}
$$

where $U_{b u s}$ is the voltage of electrical power. According to (13)-(16), the voltages of input ports of three phases can be obtained, then the voltage of central point of three phases is decided, and all the three phase voltages $v_{a}, v_{b}$ and $v_{c}$ could be acquired.

To calculate the voltage of input port of each phase, for normal BLDC control system, there are 24 different kinds of switching state which need to be considered totally. Therefore, it is a very important task to classify and determine all the data used in the model.
When the winding current is not equal to zero and PWM is 


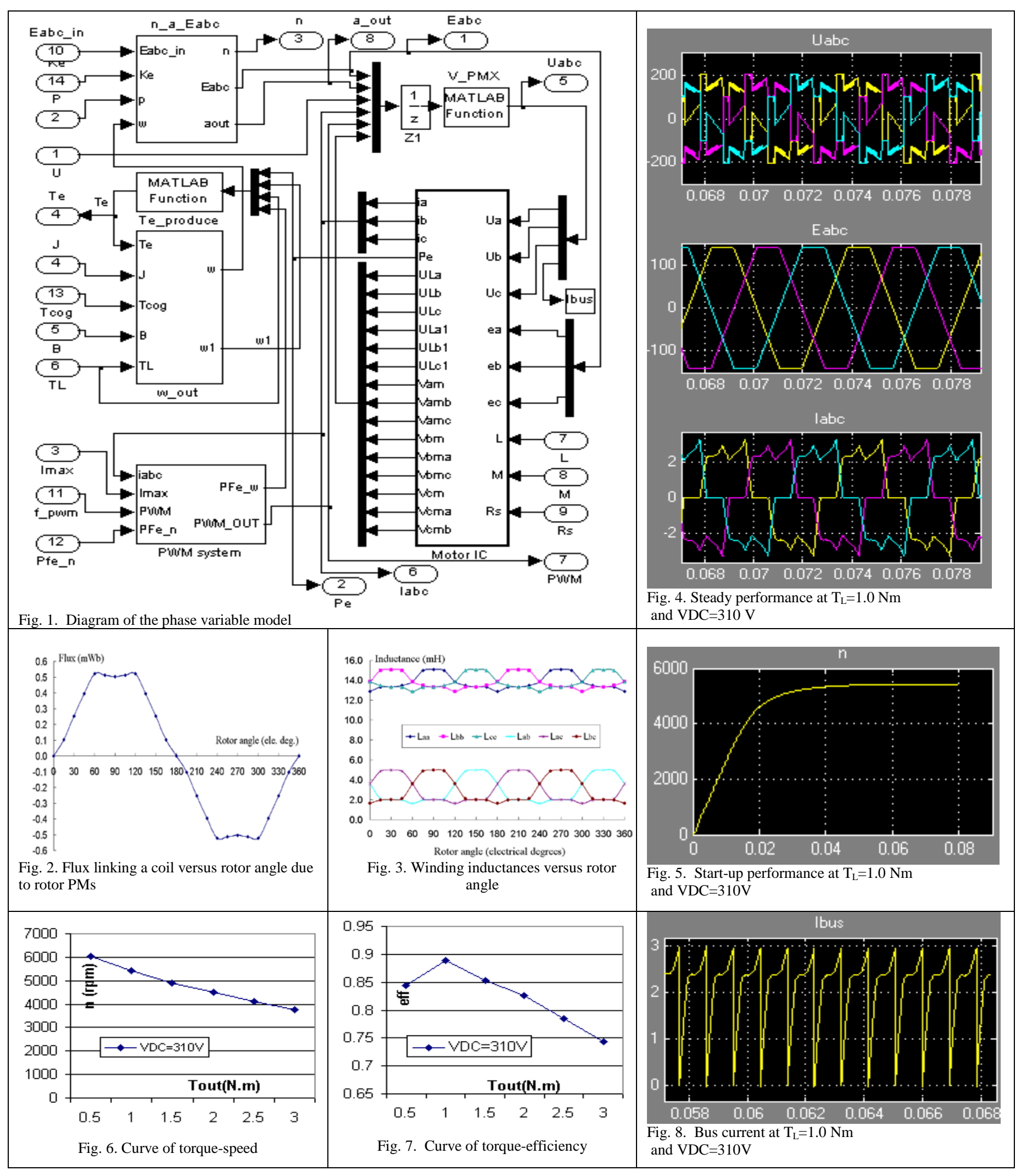




\section{SIMPLIFICATION OF THE CONNECTION OF}

EQUATION-BASED MODEL AND EXTERNAL CIRCUITS

There exist mutual inductances in (7), which will cause much difficulty to implement the mathematical model into Simulink model directly. To simplify the connection of the equation-based model and external circuits, the following definitions are used.

\section{A. Phase a:}

$$
\begin{aligned}
v_{a} & =\left(r_{a} i_{a}+L_{a a} \frac{d i_{a}}{d t}\right)+\left(L_{a b} \frac{d i_{b}}{d t}+L_{a c} \frac{d i_{c}}{d t}\right) \\
& +\left(\frac{d L_{a a}}{d \theta} i_{a}+\frac{d L_{a b}}{d \theta} i_{b}+\frac{d L_{a c}}{d \theta} i_{c}\right) \omega p+e_{a}(\theta)
\end{aligned}
$$

Supposing that

$$
\begin{aligned}
v_{a m}=\left(\frac{d L_{a a}}{d \theta} i_{a}+\frac{d L_{a b}}{d \theta}\right. & \left.i_{b}+\frac{d L_{a c}}{d \theta} i_{c}\right) \omega p \\
+ & \left(L_{a b} \frac{d i_{b}}{d t}+L_{a c} \frac{d i_{c}}{d t}\right)
\end{aligned}
$$

Then

$$
v_{a}=\left(r_{a} i_{a}+L_{a a} \frac{d i_{a}}{d t}\right)+v_{a m}+e_{a}(\theta)
$$

Supposing that

$$
v_{a}^{\prime}=v_{a}-v_{a m}
$$

Then

$$
v_{a}^{\prime}=\left(r_{a} i_{a}+L_{a a} \frac{d i_{a}}{d t}\right)+e_{a}(\theta)
$$

\section{B. Phase b:}

Similarly, the following can be obtained for phase $b$.

$$
\begin{array}{r}
v_{b m}=\left(\frac{d L_{b a}}{d \theta} i_{a}+\frac{d L_{b b}}{d \theta} i_{b}+\frac{d L_{b c}}{d \theta} i_{c}\right) \omega p \\
+\left(L_{b a} \frac{d i_{a}}{d t}+L_{b c}\right. \\
v_{b}=\left(r_{b} i_{b}+L_{b b} \frac{d i_{b}}{d t}\right)+v_{b m}+e_{b}(\theta) \\
v_{b}^{\prime}=v_{b}-v_{b m} \\
v_{b}^{\prime}=\left(r_{b} i_{b}+L_{b b} \frac{d i_{b}}{d t}\right)+e_{b}(\theta)
\end{array}
$$$$
+\left(L_{b a} \frac{d i_{a}}{d t}+L_{b c} \frac{d i_{c}}{d t}\right)
$$

\section{Phase c:}

$$
\begin{aligned}
v_{c m}=\left(\frac{d L_{c a}}{d \theta} i_{a}+\frac{d L_{c b}}{d \theta} i_{b}\right. & \left.+\frac{d L_{c c}}{d \theta} i_{c}\right) \omega p \\
& +\left(L_{c a} \frac{d i_{a}}{d t}+L_{c b} \frac{d i_{b}}{d t}\right)
\end{aligned}
$$

$v_{c}=\left(r_{c} i_{c}+L_{c c} \frac{d i_{c}}{d t}\right)+v_{c m}+e_{c}(\theta)$

$v_{c}^{\prime}=v_{c}-v_{c m}$

$v_{c}^{\prime}=\left(r_{c} i_{c}+L_{c c} \frac{d i_{c}}{d t}\right)+e_{c}(\theta)$

\section{SIMULINK MODEL AND APPLICATION}

\section{A. Simulink Implementation}

According to above equations (1)-(24), the simulation model based on a phase variable model of BLDC machine in Simulink can be obtained. Vam , Vbm and $V c m, V a, V b$ and $V c, V^{\prime} a, V^{\prime} b$ and $V^{\prime} c$ could be determined from a Matlab function based on (14)-(24). The left work of modeling the BLDC motor is similar to that of modeling a conventional DC motor, so the proposed model can be easily realized in Simulink surrounding. The completed phase variable model in Simulink surrounding is shown in Fig. 1.

\section{B. Performance Evaluation}

As an example, the rated specification of a BLDC machine is 4-pole 12-slot and $310-\mathrm{V}$. The incremental inductance $L_{a b c}$ versus rotor position and back emf versus rotor position are shown in Fig. 2 and Fig. 3, respectively. These data are obtained by nonlinear FEA. The simulation results are shown in Figs. 4-8. These results are in accordance with those of the sample motor. As the basic data which are needed by the performance evaluation can almost be obtained from this model, it can give many kinds of performance data. However, limited by paper length, only the performances concerned mostly are given here.

\section{CONCLUSION}

This paper presents the comprehensive performance evaluation of a brushless PM DC motor in dynamic conditions by an improved phase variable model based on electric-magnetic field coupling with its external circuits. A mathematical method, which can apply the equation-based model to simulation model directly, is introduced in detail. By running this model, the comprehensive performance evaluation of the motor could be obtained. 


\section{REFERENCES}

[1] J T. Kenjo, Permanent Magnet and Brushless DC Motors, Oxford University Press, 1985.

[2] S. L. Ho, W. N. Fu, H. L. Li, H. C. Wong, and H. Tan, "Performance analysis of brushless dc motors including features of the control loop in the finite element modeling," IEEE Trans. Magn., Vol. 37, No. 5, pp. 3370-3374, Sept. 2001.

[3] O. A. Mohammed, S. Liu, and Z. Liu, "A phase variable model of brushless dc motors based on finite element analysis and its coupling with external circuits,” IEEE Trans. Magn., Vol. 41, No. 5, pp. 1576-1579, May 2005.
[4] J. F Gieras, "Analytical approach to cogging torque calculation of PM brushless motors,” IEEE Trans. Industry Applications, Vol. 40, No. 5, pp. 1310-1316, Sept./Oct. 2004.

[5] M. Gyimesi and D. Ostergaard, "Inductance computation by incremental finite element analysis,” IEEE Trans. Magn., Vol. 35, No. 3, pp. 1119-1122, May 1999.

[6] Y. G. Guo, J. G. Zhu, H. W. Lu, R. Chandru, S. H. Wang, and J. X. Jin, "Determination of winding inductance in a claw pole permanent magnet motor with soft magnetic composite core," in Proc. Australasian Universities Power Eng. Conf., Hobart, Australia, Sept. 25-28, 2005, pp. 491-496. 